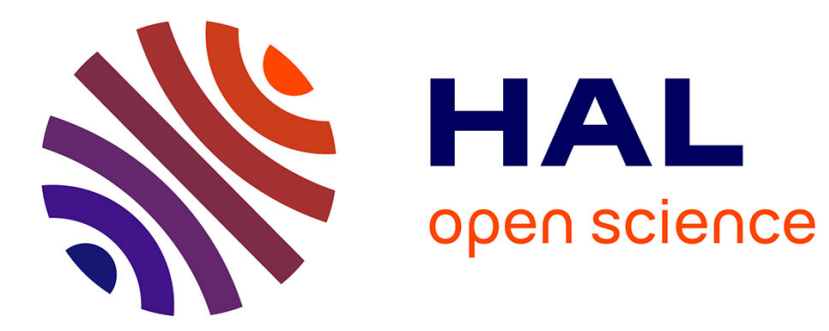

\title{
Determination of Q-convex bodies by X-rays
}

\author{
Sara Brunetti, Alain Daurat
}

\section{To cite this version:}

Sara Brunetti, Alain Daurat. Determination of Q-convex bodies by X-rays. Proceedings of the Workshop on Discrete Tomography and its Applications, 2005, New-York, United States. pp.67-81, 10.1016/j.endm.2005.05.056 . hal-00023089

\section{HAL Id: hal-00023089 \\ https://hal.science/hal-00023089}

Submitted on 20 Apr 2006

HAL is a multi-disciplinary open access archive for the deposit and dissemination of scientific research documents, whether they are published or not. The documents may come from teaching and research institutions in France or abroad, or from public or private research centers.
L'archive ouverte pluridisciplinaire HAL, est destinée au dépôt et à la diffusion de documents scientifiques de niveau recherche, publiés ou non, émanant des établissements d'enseignement et de recherche français ou étrangers, des laboratoires publics ou privés. 


\title{
Determination of Q-convex bodies by X-rays
}

\author{
Sara Brunetti ${ }^{1}$ \\ Dipartimento di Scienze Matematiche e Informatiche, Università di Siena, Pian \\ dei Mantellini 44, 53100, Siena, Italy
}

Alain Daurat ${ }^{2,3}$

LSIIT UMR 7005 CNRS-ULP, Pôle API, Boulevard Sébastien Brant, 67400

Illkirch-Graffenstaden, France

\begin{abstract}
The class of Q-convex bodies is defined, and the uniqueness result proved by Gardner and McMullen in 1980 for planar convex bodies is extended to this new class.
\end{abstract}

Keywords: Convex Bodies, Geometric Tomography, Uniqueness

\section{Introduction}

Let $f$ be a density function from the euclidean plane $\mathbb{E}^{2}$ in $\mathbb{R}$. The X-ray of $f$ in a direction $p$ is the function assigning to each straight line parallel to $p$ the integral of $f$ over the line (when it exists). In this paper we consider the problem of the determination of an unknown density function in the restricted class of characteristic functions of subsets of $\mathbb{E}^{2}$. Hence this subject fits in the

$\overline{1}$ Email: sara.brunetti@unisi.it

2 Email: daurat@dpt-info.u-strasbg.fr

3 Partially supported by the Project IRMC-EPML-9 of French CNRS 
intersection of discrete and geometric tomography. (We recommend [11] and [6] as comprehensive books on these areas.) It is well known that X-rays data in a finite number of directions do not permit the determination of all subsets of $\mathbb{E}^{2}$. An interesting question proposed by Hammer is how many $\mathrm{X}$ rays one needs to exactly reconstruct a convex body. There are some papers that address: the related problem of the existence of a set of directions which uniquely determine the convex body [8,9]; the approximative reconstruction of a convex body [12]; the reconstruction of boundary points of convex body $[6,14]$; the stability of the reconstruction [13]. As far as we know, none of these results provide a reconstruction algorithm which gives a complete solution to any desired accuracy. (see also note 1.2 of [6])

In the discrete case similar results hold for convex lattice sets, and moreover a reconstruction algorithm is known [1] based on a new class of sets, called the Q-convex lattice sets. Recently, the authors of this paper have proved that a convex body is arbitrary close to a convex lattice set whose X-rays are close enough to the X-rays of the convex body [2]. This result would permit the reconstruction of convex bodies through their approximative lattice convex sets. Unfortunately to carry out the reconstruction we should have an algorithm solving the problem from approximative X-rays, and we have not. What we have is a reconstruction algorithm from approximative X-rays for Qconvex lattice sets [3]. In this paper we extend this notion to the continuous setting and we hope this could help to produce a more efficient algorithm. This class is more general than the class of convex bodies: while a convex body is the closure of the convex hull of its exposed points (at which an hyperplane supports the body), a Q-convex body is the closure of the Q-convex hull of its "salient" points (at which an "almost semi-plane" supports the body). In particular we study the uniqueness of Q-convex bodies from their X-rays in a suitable set of directions. This result represents the first step in the direction of answering Hammer's question for Q-convex bodies.

\section{Q-convexity and Basic Properties}

There are some basic notions of convexity that can be generalized to Qconvexity. We start with some basic definitions and then we proceed by showing some properties.

In this paper a direction is given by its equation $p(M)=a x_{M}+b y_{M}$. For each point $M=(p(M), q(M)) \in \mathbb{E}^{2}$ the four quadrants around $M$ determined 
by the set of directions $\mathcal{D}=\{p, q\}$ are defined by the following formulas:

$$
\begin{aligned}
& R_{0}^{p q}(M)=\left\{(p(N), q(N)) \in \mathbb{E}^{2} / p(N) \leq p(M) \text { and } q(N) \leq q(M)\right\}, \\
& R_{1}^{p q}(M)=\left\{(p(N), q(N)) \in \mathbb{E}^{2} / p(N) \geq p(M) \text { and } q(N) \leq q(M)\right\}, \\
& R_{2}^{p q}(M)=\left\{(p(N), q(N)) \in \mathbb{E}^{2} / p(N) \geq p(M) \text { and } q(N) \geq q(M)\right\}, \\
& R_{3}^{p q}(M)=\left\{(p(N), q(N)) \in \mathbb{E}^{2} / p(N) \leq p(M) \text { and } q(N) \geq q(M)\right\} .
\end{aligned}
$$

A body is a compact subset of $\mathbb{E}^{2}$ which is the closure of its interior.

Definition 2.1 A body $F$ is Q-convex with respect to $\mathcal{D}=\{p, q\}$, if $R_{k}^{p q}(M) \cap$ $F \neq \emptyset$ for all $k \in\{0,1,2,3\}$ implies $M \in F$.

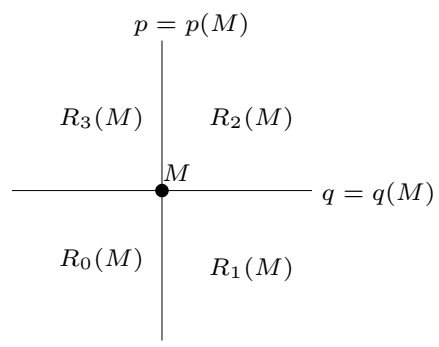

(a)

Fig. 1. The four quadrants for the coordinate directions $p=x$ and $q=y$

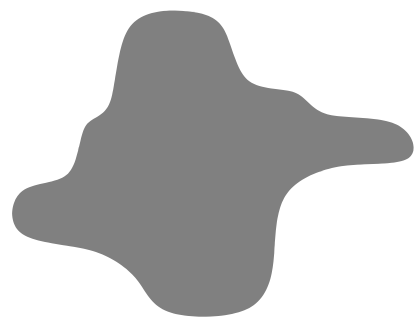

(a)

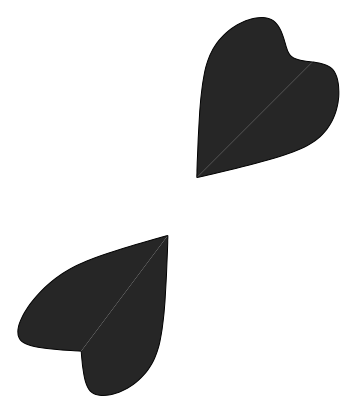

(b)

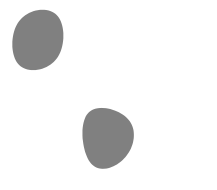

(c)

Fig. 2. Q-convex bodies (a,b), and a not-Q-convex body (c) for the coordinate directions $p=x$ and $q=y$

Definition 2.2 Let $\mathcal{D}$ be any finite set of directions. A body is Q-convex w.r.t. $\mathcal{D}$, if it is a $\mathrm{Q}$-convex body w.r.t. any pair of directions in $\mathcal{D}$. 
Definition 2.3 An ASP (almost-semi-plane) w.r.t. $\mathcal{D}$ is a quadrant $R_{i}^{p q}(M)$ with $p, q \in \mathcal{D}$ such that for every direction $r \in \mathcal{D}$ a semi-line of direction $r$ with starting point $M$ is contained in $R_{i}^{p q}(M)$.

The property for $R_{i}^{p q}(M)$ to be an ASP does not depend on the point $M$. We denote by $\mathcal{A}_{\mathcal{D}}$ the set of indexes $(i, p, q)$ such that $R_{i}^{p q}(M)$ is an ASP.

An ASP is a maximal member ordered by inclusion.

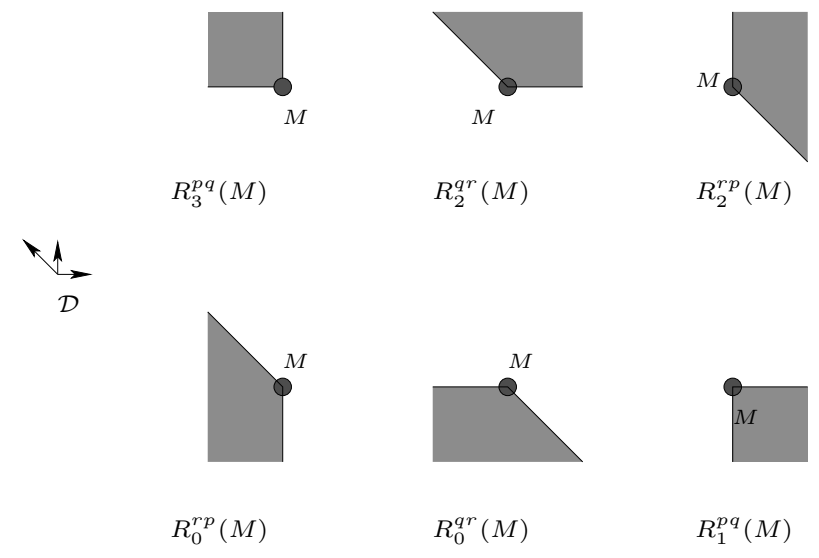

Fig. 3. The ASP for $\mathcal{D}=\{p, q, r\}$ with $p=x, q=y, r=x+y$.

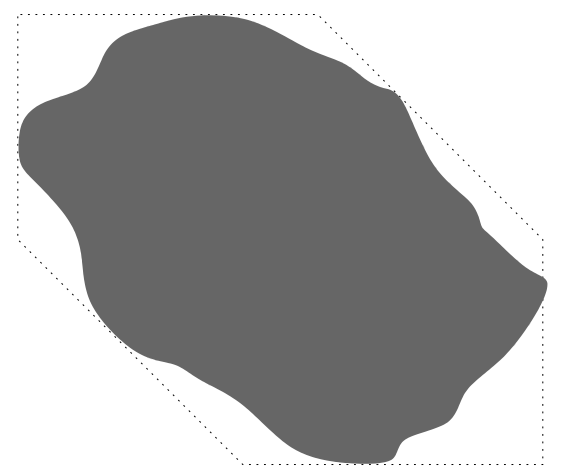

Fig. 4. A Q-convex set for $\mathcal{D}=\{p, q, r\}$ with $p=x, q=y, r=x+y$.

Q-convexity is very similar to convexity where the semi-planes replaced by the ASPs. As the intersection of Q-convex sets is also a Q-convex sets the Q-convex hull of a set $F$ can be defined as:

Definition 2.4 The Q-convex hull of a set $F$, denoted by $\operatorname{Qconv}(F)$ is the intersection of all the Q-convex sets containing $F$. 
Property 2.5 The Q-convex hull of a set F satisfies

$$
\operatorname{Qconv}(F)=\left\{M \in \mathbb{E}^{2}: \forall(i, p, q) \in \mathcal{A}_{\mathcal{D}}, R_{i}^{p q}(M) \cap F \neq \emptyset\right\}
$$

If $p$ and $q$ are two lattice directions, we denote by $\langle i, j\rangle_{p, q}$ (and even $\langle i, j\rangle$ if there is no ambiguity) the point $M$ which satisfies $p(M)=i$ and $q(M)=j$.

Proof. Let $F$ be a set, and $G=\left\{M \in \mathbb{E}^{2}: \forall(i, p, q) \in \mathcal{A}_{\mathcal{D}}, R_{i}^{p q}(M) \cap F \neq \emptyset\right\}$. We have that $G \subset F$.

Let $M$ be a point which is not in $G$, then there exists an ASP, $R_{i}^{p q}(M)$, which does not contain any point of $F$. So by definition of $G$, it also does not contain any point of $G$. As the ASP $R_{i}^{p q}(M)$ contains a quadrant along any couple of directions in $\mathcal{D}$, this proves that for any point $M \notin G$ and any directions $p, q \in \mathcal{D}$ holds that $R_{i}^{p q}(M) \cap G=\emptyset$. So $G$ is a Q-convex set containing $F$. We deduce $\operatorname{Qconv}(F) \subseteq G$.

We are going to prove that $G \subseteq \mathrm{Q} \operatorname{conv}(F)$. If $\mathcal{D}$ has only two directions it is clear, so we suppose that $\operatorname{card}(\mathcal{D}) \geq 3$. We suppose that there exists a point $M$ in $G \backslash$ Qconv $(F)$. Let $R_{i}^{p q}(M)$ be a quadrant which contains no point of $F$ and which is maximum for the following order:

$$
R_{i}^{p q}(M) \prec R_{j}^{r s}(M) \text { iff } R_{i}^{p q}(M) \subseteq R_{j}^{r s}(M) \text { or } R_{i+2}^{p q}(M) \subseteq R_{j}^{r s}(M)
$$

By replacing if necessary $p, q$ by $-p,-q$, we can suppose that $i=0$. The quadrant $R_{i}^{p q}(M)$ is not an ASP because $M \in G$. So there exists a direction $r$ such that $r=\alpha p+\beta q$ with $\alpha \beta>0$. By possibly replacing $r$ by $-r$, we can suppose that $\alpha>0, \beta>0$. By maximality of $R_{0}^{p q}(M)$ : $i$ ) the quadrants $R_{1}^{q r}(M) \subset R_{0}^{p r}(M)$ and $R_{1}^{p r} \subset R_{0}^{q r}(M)$ contain a point of $F$, and $i i$ ) the quadrant $R_{2}^{q r}(M)$ contains a point of $F$. By Q-convexity w.r.t the directions q, $r, M \notin \operatorname{Qconv}(F)$ implies that $R_{3}^{q r}(M)$ does not contain any point of $F$. Similarly, $R_{3}^{p r}(M)$ does not contain any point of $F$. As $R_{2}^{q r}(M)=R_{3}^{p r}(M) \cup$ $R_{2}^{p q}(M)$, the quadrant $R_{2}^{p q}(M)$ contains a point of $F$. Let $A \in R_{1}^{q r}(M) \cap F$, $B \in R_{1}^{p r}(M), C \in R_{2}^{p q}(M)$, and $N=\langle\min (p(B), p(C)), q(M)\rangle_{p, q}$. The four $p, q$-quadrants around $N$ contain $A$ or $B$ or $C$, and so $N \in F$ in contradiction with $R_{3}^{q r}(M) \cap F=\emptyset$. The thesis follows.

Remark 2.6 In the discrete plane $\mathbb{Z}^{2}$, there are two notions of Q-convexity ("normal" Q-convexity and strong Q-convexity [1,3]) when we consider a set $\mathcal{D}$ of more than two directions, because there is no discrete analogue to Property 2.5 .

The notion of exposed point can be generalized by that of salient point. 
Definition 2.7 A salient point is one $M$ of $F$ such that $M \notin \mathrm{Q} \operatorname{conv}(F \backslash M)$.

Property 2.8 Here we list some properties that we can deduce from the definitions.

- Any point $M$ of $F$ is salient if and only if $(i, p, q) \in \mathcal{A}_{\mathcal{D}}$ exists such that $R_{i}^{p q}(M)$ is a supporting $A S P$ at $M$, which means $R_{i}^{p q}(M) \cap F=\{M\}$.

- Any Q-convex compact set $F$ is the Q-convex hull of its salient points.

Proof.

- The first part of the property is a simple consequence of Property 2.5.

- Let $M \in \operatorname{Qconv}(F)$. We must prove that each ASP around $M$ contains a salient point. So we consider an ASP $R_{i}^{p q}(M)$. We can suppose that $i=0$. As $F$ is compact, there is a point $N \in F \cap R_{i}^{p q}(M)$ which minimizes $p(N)+q(N)$, the quadrant $R_{0}^{p q}(N)$ is supporting $F$, so $N$ is a salient point.

It must be noticed that Q-convexity is very linked with restrictedorientation-convexity. We recall that a set is restricted-orientation-convex w.r.t. $\mathcal{D}$ if its intersection with any line parallel to $\mathcal{D}$ is empty or connected (see $[5])$.

Property 2.9 Let $E$ be a subset of $\mathbb{E}^{2}$.

- If $E$ is $Q$-convex w.r.t. $\mathcal{D}$, then it is restricted-orientation-convex w.r.t. $\mathcal{D}$.

- If $E$ is restricted-orientation-convex w.r.t. $\mathcal{D}$ and connected, then $E$ is Q-convex w.r.t. $\mathcal{D}$.

The inverse of these two assertions is false (see Figures 2(c) and 2(b)).

\section{Proof.}

- Let $p$ be a direction of $\mathcal{D}$, and $A$ and $B$ be two points of $E$ such that $p(A)=p(B)$. For every point in the segment $[A B]$, its four $p, q$-quadrants contain either $A$ or $B$ so that the point belongs to $E$.

- Let $p, q$ be two directions of $\mathcal{D}$ and $M$ be any point such that all the $p, q$ quadrants around $M$ contain a point of $E$. We are going to show that $M \in E$. Let $l_{i}$ denote the semi-line $R_{i}^{p q}(M) \cap R_{(i+1) \bmod 4}^{p q}(M)$. If $l_{3} \cup l_{0}$ does not contain any point of $E$, then the two sets $E \cap R_{0}^{p q}(M)$ and $E \backslash R_{0}^{p q}(M)$ are open relatively to $E$ and non-empty which is in contradiction with the connectivity of $E$. So $l_{3} \cup l_{0}$ contains a point of $E$. Similarly we can prove that $l_{i} \cup l_{i+1}$ contain a point of $E$ for $i=0,1,2$. If $l_{0}$ and $l_{2}$ contain a point of $E$ then $M$ is in $E$ otherwise $l_{1}$ and $l_{3}$ contain a point $E$ and so $M$ is also 
in $E$.

\section{Uniqueness result for Q-convex bodies}

Definition 3.1 The X-ray $X_{p} F$ of a Q-convex body $F$ in direction $p$ is the function giving the length of each line segment of $F$ parallel to $p: X_{p} F(k)=$ length $(F \cap\{p(M)=k\})$.

A set of directions $\mathcal{D}$ determines a class of sets if for any set of this class, there does not exist any other set of this class which has the same X-rays in $\mathcal{D}$.

The main result of this paper concerns the existence of a set of directions $\mathcal{D}$ which determines the Q-convex bodies w.r.t. $\mathcal{D}$ :

Theorem 3.2 Let $\mathcal{D}$ be a set of directions. The two following assertions are equivalent:

(a) $\mathcal{D}$ determines the $Q$-convex bodies w.r.t. $\mathcal{D}$.

(b) $\mathcal{D}$ determines the convex bodies.

So a set of four directions with a transcendental cross-ratio ([8, Theorem $4])$ or the set of directions $\{(1,0),(0,1),(2,1),(-1,2)\}([7$, Theorem 6.2]) determine the Q-convex bodies w.r.t. the same set of directions.

The rest of the section is devoted to the proof of this theorem that follows the ideas and schemes of the proofs in $[4,7,8]$,

First we need to recall some definitions:

Definition 3.3 Let $\mathcal{D}$ be a set of directions.

- A $\mathcal{D}$-polygon $P$ is a convex polygon such that any line of direction in $\mathcal{D}$ contains zero or two vertices of $P$.

- A $\mathcal{D}$-sequence is a sequence $\left(A_{k}\right)_{k \in \mathbb{F}_{m}}$ of $m$ points of $\mathbb{E}^{2}$ such that $m$ is even and for any $p$ in $\mathcal{D}$ there is an $s \in \mathbb{F}_{m}$ such that

$$
\begin{array}{ccc}
p\left(A_{s-1}\right)<p\left(A_{s-2}\right) & <\cdots<p\left(A_{s-\frac{m}{2}}\right) \\
\text { । } & \text { । } & \text { । } \\
p\left(A_{s}\right) & <p\left(A_{s+1}\right)<\cdots<p\left(A_{s+\frac{m}{2}-1}\right)
\end{array}
$$

Theorem 3.2 can be precised as follows: 
Theorem 3.4 Let $\mathcal{D}$ be a set of directions. The following assertions are equivalent:

(i) $\mathcal{D}$ does not determine the convex bodies.

(ii) There exists a $\mathcal{D}$-polygon.

(iii) $\mathcal{D}$ does not determine the Q-convex bodies w.r.t. $\mathcal{D}$.

(iv) There exists a $\mathcal{D}$-sequence.

The equivalence (i) $\Leftrightarrow$ (ii) is in [8], [7, theorem 6.1]. The implication (iv) $\Rightarrow$ (ii) is an extension of [4, Lemmas 27,28$]$ to any directions, since the proof does use the fact that only lattice-directions are considered. The implication (i) $\Rightarrow$ (iii) is clear because every convex body is Q-convex along any set of directions, (ii) $\Rightarrow$ (iv) is also clear since any $\mathcal{D}$-polygon is a $\mathcal{D}$-sequence. So we only have to prove (iii) $\Rightarrow$ (iv).

In the following we suppose that case (iii) arises.

Let $F^{+}$and $F^{-}$be two Q-convex bodies having same X-rays in the directions of $\mathcal{D}$. We define $E^{+}=\operatorname{interior}\left(F^{+}\right) \backslash F^{-}$and $E^{-}=\operatorname{interior}\left(F^{-}\right) \backslash F^{+}$. So, $E^{+}$and $E^{-}$are open.

For any point $M \in E^{+}$and any direction $p \in \mathcal{D}$, the set $E^{+} \cap\{p=p(M)\}$ is an open interval (by Q-convexity of $F^{+}$and $F^{-}$) and $X_{p} E^{+}(p(M))>0$. Since $X_{p} E^{+}=X_{p} E^{-}, E^{-} \cap\{p=p(M)\}$ is an interval which is a translate of $E^{+} \cap\{p=p(M)\}$. We shall denote the image of $M$ by such a translation by $M_{p} \in E^{-}$.

We will need the following order (extension of an order by inclusion) among the quadrants:

$$
R_{i}^{p q}(M)<R_{j}^{r s}\left(M^{\prime}\right) \text { iff } R_{i}^{p q}(O) \subseteq R_{j}^{r s}(O)
$$

where $M, M^{\prime}$ and $O=(0,0)$ are points and $p, q, r, s$ are directions in $\mathcal{D}$.

The area of a measurable subset $E$ is the Lebesgue measure of $E$ and is denoted $\lambda(E)$.

In the following we shall list some lemmas.

Lemma 3.5 For every point $M \in E^{+}$there exists one and only one $(i, p, q) \in$ $\mathcal{A}_{\mathcal{D}}$ such that $R_{i}^{p q}(M) \cap E^{-}=\emptyset$.

For the sake of simplicity, here (and in the following) we state the lemma for $M \in E^{+}$but the lemma holds exchanging $E^{+}$and $E^{-}$.

Proof. Since $F^{-}$is Q-convex and $M \notin F^{-}$, a quadrant exists having empty intersection with $E^{-}$. Let us consider $R_{i}^{p q}(M)$ the maximal quadrant w.r.t. to 
the order (1) having empty intersection with $E^{-}$, and suppose that $(i, p, q) \notin$ $\mathcal{A}_{\mathcal{D}}$. Let us assume without lost of generality that $i=0$ : by $(0, p, q) \notin \mathcal{A}_{\mathcal{D}}$ follows that a direction $r \in \mathcal{D}$ exists such that the straight line parallel to $r$ through $M$ intersects $R_{0}^{p q}(M)$ into $M$ only, and that $p(M)<p\left(M_{q}\right)$ and $q(M)<q\left(M_{p}\right)$. Consider $M_{r} \in E^{-}$:

- if $p\left(M_{r}\right)<p(M)$, then $R_{1}^{p r}(M) \cap E^{-}=\emptyset$ because otherwise a point $N$ exists such that $M \in \mathrm{Qconv}_{p r}\left(M_{r}, N, M_{p}\right)$ but $M \in E^{+}$.

- if $p\left(M_{r}\right)>p(M)$, then $R_{3}^{q r}(M) \cap E^{-}=\emptyset$ because otherwise a point $N$ exists such that $M \in \mathrm{Q}^{\operatorname{conv}_{q r}}\left(M_{r}, N, M_{q}\right)$ but $M \in E^{+}$.

Both cases contradict the maximality of $R_{0}^{p q}(M)$ w.r.t. inclusion. Therefore we conclude that $(0, p, q) \in \mathcal{A}_{\mathcal{D}}$.

Suppose there are two ASP having empty intersection with $E^{-}$. Then, they contain a straight line through $M$ parallel to a direction $r$ in $\mathcal{D}$ contradicting the fact that $F^{+}$and $F^{-}$have same X-rays in direction $r$.

Let $E_{i p q}^{+} \subset E^{+}$whose points $M$ satisfy $R_{i}^{p q}(M) \cap E^{-}=\emptyset$ and $E_{i p q}^{-} \subset E^{-}$ whose points $M$ satisfy $R_{i}^{p q}(M) \cap E^{+}=\emptyset$, for any $(i, p, q) \in \mathcal{A}_{\mathcal{D}}$. We can partition $E^{+}$and $E^{-}$as:

$$
E^{+}=\cup_{(i, p, q) \in \mathcal{A}_{\mathcal{D}}} E_{i p q}^{+}, \quad E^{-}=\cup_{(i, p, q) \in \mathcal{A}_{\mathcal{D}}} E_{i p q}^{-} .
$$

Additionally:

$$
B_{p q}(C, \varepsilon)=\left\{M \in \mathbb{E}^{2}:|p(M)-p(C)|<\varepsilon \text { and }|q(M)-q(C)|<\varepsilon\right\} .
$$

Lemma 3.6 $E_{i p q}^{+}$is open.

Proof. Let $C$ be a point in $E_{i p q}^{+}$and suppose $i=0$. We have that $q\left(C_{p}\right)>$ $q(C)$ and $p\left(C_{q}\right)>p(C)$. Since $E^{+}$is open, there exists $\varepsilon$ such that the ball $B_{p q}(C, \varepsilon)$ is included in $E^{+}$. Let $M$ be any point in $B_{p q}(C, \varepsilon)$. We show that $M$ is in $E_{i p q}^{+}$too. This immediately follows if $p\left(M_{q}\right)>p(M)$ and $q\left(M_{p}\right)>q(M)$.

Suppose that $p\left(M_{q}\right)<p(M)$ (analogously, for the case $q\left(M_{p}\right)<q(M)$ ). As $M_{q} \notin R_{0}^{p q}(C)$ we have $q(M)>q(C)$ and then $\langle p(C), q(M)\rangle \in$ Qconv $_{p q}\left(M_{q}, C_{p}, C_{q}\right)$ contradicting that $B_{p q}(C, \varepsilon)$ is included in $E^{+}$.

This concludes the proof.

Definition 3.7 Two points $A, B \in E_{i p q}^{+}$are equivalent if a point $N_{A B}$ exists such that $A, B \in R_{i}^{p q}\left(N_{A B}\right) \in \mathcal{A}_{\mathcal{D}}$ and $R_{i}^{p q}\left(N_{A B}\right) \cap E^{-}=\emptyset$. (We write $A \sim B$ ). 


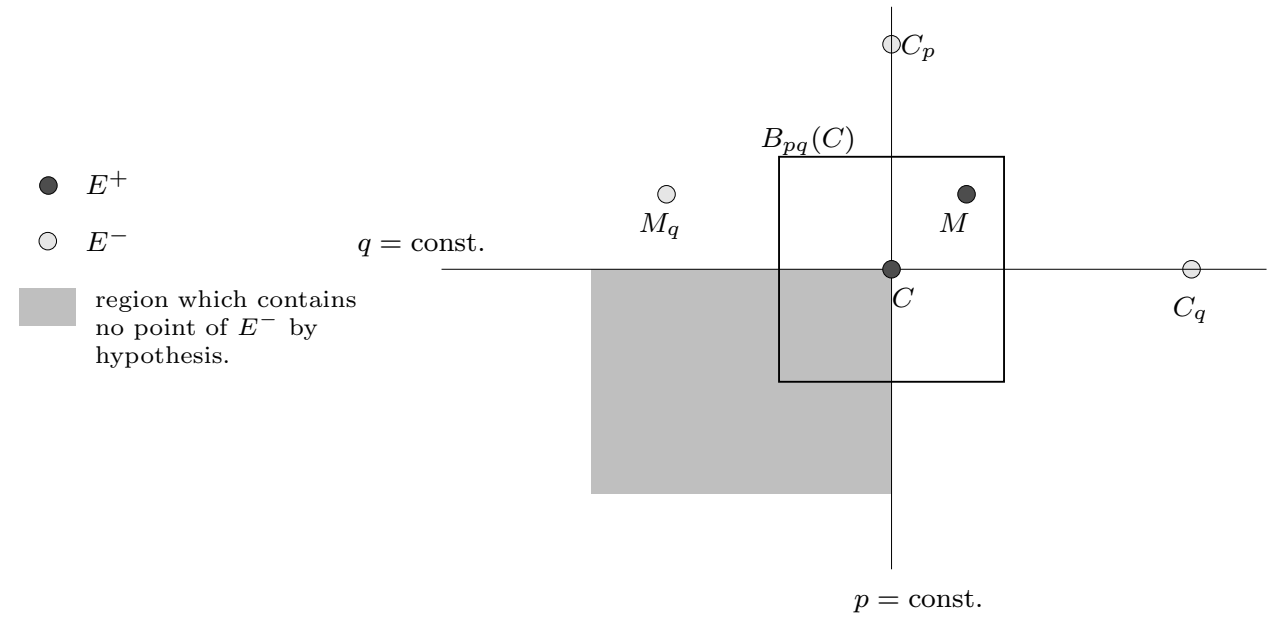

Fig. 5. Illustration for Lemma 3.6

Notice that if $A, B \in E_{0 p q}^{+}$we have to look at $N_{A B}=$ $\langle\max (p(A), p(B)), \max (q(A), q(B))\rangle$. (Analogously, for a different value of $i$.)

Lemma $3.8 \sim$ is an equivalence relation on $E^{+}$.

Proof. We shall prove that if $A, B, C \in E_{i p q}^{+}$, and $A \sim B$ and $B \sim C$, then

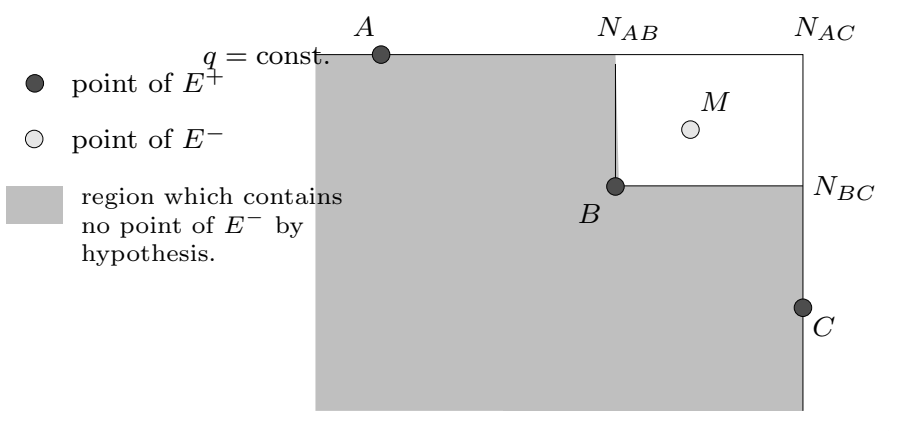

Fig. 6. Illustration for Lemma 3.8

$A \sim C$. Without lost of generality we suppose $i=0$. Thus we have to show that $R_{0}^{p q}\left(N_{A B}\right) \cap E^{-}=\emptyset$ and $R_{0}^{p q}\left(N_{B C}\right) \cap E^{-}=\emptyset$ imply $R_{0}^{p q}\left(N_{A C}\right) \cap E^{-}=\emptyset$, where $N_{A C}=\left\langle\max \left(p\left(N_{A B}\right), p\left(N_{B C}\right)\right), \max \left(q\left(N_{A B}\right), q\left(N_{B C}\right)\right)\right\rangle$. When $N_{A C}$ is equal to $N_{A B}$ or $N_{B C}$, the wished claim is clear. So we can suppose the case in which $q(A)>q(B)>q(C), p(C)>p(B)>p(A)$ and hence $q\left(N_{A B}\right) \geq q\left(N_{B C}\right)$ and $p\left(N_{A B}\right) \leq p\left(N_{B C}\right)$.

Suppose by contradiction that $R_{0}^{p q}\left(N_{A C}\right) \cap E^{-} \neq \emptyset$. Since $E^{+}$is open, 
$\lambda\left(R_{0}^{p q}(B) \cap E^{+}\right)=\mu>0$. Let us choose $M \in E^{-} \cap R_{0}^{p q}\left(N_{A C}\right)$ such that $\lambda\left(R_{0}^{p q}(M) \cap E^{-}\right)<\frac{\mu}{2}$. We have that $p(B)<p(M)<p(C)$ and $q(A)>$ $q(M)>q(B)$ and hence $R_{0}^{p q}(B) \subset R_{0}^{p q}(M)$. Since $F^{+}$and $F^{-}$have the same $\mathrm{X}$-rays in directions $p, q$, we deduce that

$$
\lambda\left(R_{0}^{p q}(M) \cap E^{+}\right)+\lambda\left(R_{1}^{p q}(M) \cap E^{+}\right)=\lambda\left(R_{0}^{p q}(M) \cap E^{-}\right)+\lambda\left(R_{1}^{p q}(M) \cap E^{-}\right)
$$

and

$$
\lambda\left(R_{2}^{p q}(M) \cap E^{+}\right)+\lambda\left(R_{1}^{p q}(M) \cap E^{+}\right)=\lambda\left(R_{2}^{p q}(M) \cap E^{-}\right)+\lambda\left(R_{1}^{p q}(M) \cap E^{-}\right) .
$$

Thus, we obtain

$$
\lambda\left(R_{2}^{p q}(M) \cap E^{+}\right)=\lambda\left(R_{2}^{p q}(M) \cap E^{-}\right)+\lambda\left(R_{0}^{p q}(M) \cap E^{+}\right)-\lambda\left(R_{0}^{p q}(M) \cap E^{-}\right)>\mu / 2 .
$$

Since every quadrant of $M \in E^{-}$has non-empty intersection with $E^{+}$, we get a contradiction.

By Lemma 3.6 follows that:

Lemma 3.9 Any equivalence class $C$ is open.

For every class $C$, there exist $(i, p, q) \in \mathcal{A}_{\mathcal{D}}$, and a point $N$ such that $C=\left(E^{+} \cup E^{-}\right) \cap R_{i}^{p q}(N)$.

Lemma 3.10 If $A, B \in E^{+}$and a point $N_{A B}$ exists such that $A, B \in$ $R_{i}^{p q}\left(N_{A B}\right)$ and $R_{i}^{p q}\left(N_{A B}\right) \cap E^{-}=\emptyset$, then $A \sim B$.

Proof. Let $(i, p, q)$ be maximal w.r.t. the order (1). Assume $i=0$ and then $N_{A B}=\langle\max (p(A), p(B)), \max (q(A), q(B))\rangle$. If $(0, p, q) \notin \mathcal{A}_{\mathcal{D}}$, then a direction $r \in \mathcal{D}$ exists such that the straight line parallel to $r$ through $N_{A B}$ intersects $R_{0}^{p q}\left(N_{A B}\right)$ in $N_{A B}$ only. Moreover we can suppose that $r=\alpha p+\beta q$ with $\alpha, \beta>0$.

If $N_{A B}=A$ or $N_{A B}=B$ the proof follows the same considerations as those exhibited in the proof of Lemma 3.5.

Otherwise two cases can arise: $N_{A B}=\langle p(B), q(A)\rangle$ and $N_{A B}=$ $\langle p(A), q(B)\rangle$. Let us consider the first one. If $p(A)>p\left(A_{r}\right)$ and $p(B)<p\left(B_{r}\right)$, or $p(A)<p\left(A_{r}\right)$ and $p(B)>p\left(B_{r}\right)$ :

- If $r(A) \leq r(B)$, then $B \in \mathrm{Q}^{\operatorname{conv}}{ }_{p r}\left(B_{r}, B_{p}, A_{r}\right)$ contradicting that $B \in E^{+}$.

- If $r(A) \geq r(B)$, then $A \in \mathrm{Q} \operatorname{conv}_{q r}\left(A_{r}, A_{q}, B_{r}\right)$ contradicting that $A \in E^{+}$.

Therefore we deduce that $\left(R_{0}^{p r}(A) \cup R_{0}^{p r}(B)\right) \cap E^{-}=\emptyset$ or $\left(R_{0}^{q r}(A) \cup R_{0}^{q r}(B)\right) \cap$ $E^{-}=\emptyset$. In the first case, take $N_{A B}^{\prime}=\langle\max (p(A), p(B)), \max (r(A), r(B))\rangle_{p, r}$ : 
$R_{0}^{p r}\left(N_{A B}^{\prime}\right) \cap E^{-}=\emptyset$ contradicts that $(0, p, q)$ is maximal w.r.t. the order $(1)$. Second case is completely similar.

Lemma 3.11 Let $A$ and $B$ be two points of $E_{i p q}^{+}$such that $A \sim B$. For every point $M$ of $E^{+}$such that $r(A) \leq r(M) \leq r(B), r \in \mathcal{D}$, there holds that $M \sim A \sim B$.

Proof. Let us suppose $i=0$. If $M \in R_{0}^{p q}\left(N_{A B}\right)$, the thesis immediately follows. Hence we study the other case. By Q-convexity it follows that $p\left(M_{r}\right)>p(M)$ (or equivalently $q\left(M_{r}\right)>q(M)$ ). If $p(M)>\max (p(A), p(B))$, as $q(M) \geq \min (q(A), q(B))$, we have $A \in R_{0}^{p q}(M)$ or $B \in R_{0}^{p q}(M)$. Similarly if $q(M)>\max \left(q(A), q(B)\right.$ then we have also $A \in R_{0}^{p q}(M)$ or $B \in R_{0}^{p q}(M)$.

In order to conclude we have to show that $R_{0}^{p q}(M) \cap E^{-}=\emptyset$ or, equivalently, that $p\left(M_{q}\right)>p(M)$ and $q\left(M_{p}\right)>q(M)$. Indeed if $q\left(M_{p}\right)<q(M)$ then $M \in \operatorname{Qconv}_{p r}\left(M_{p}, M_{r}, A_{p}, B_{p}\right)$ and if $p\left(M_{q}\right)<p(M)$ then $M \in$ Qconv $_{q r}\left(M_{q}, M_{r}, A_{q}, B_{q}\right)$.

Remark 3.12 In particular, the lemma states that for any line $\{r=r(M)\}$ parallel to $r \in \mathcal{D}$ intersecting an equivalence class $C$ in $E_{i p q}^{+},\{r=r(M)\} \cap$ $\left(E^{+} \backslash C\right)=\emptyset$.

Lemma 3.13 Let $r \in \mathcal{D}$. If $A \sim B$, then $A_{r} \sim B_{r}$.

Proof. Suppose that $A, B \in E_{0 p q}^{+}$and $r=\alpha p+\beta q, \alpha \leq 0$ and $\beta \geq 0$, and $r(A)<r(B)$.

Let $i, j$ such that $R_{i}^{p q}\left(A_{r}\right) \cap E^{+}=\emptyset$ and $R_{j}^{p q}\left(B_{r}\right) \cap E^{+}=\emptyset$.

We can prove that $i=j$ by considering all the cases. For example in the case $i=2, j=1$, then $B_{r p} \in E^{+} \cap R_{2}^{p q}\left(A_{r}\right)$ or $A_{r p} \in E^{+} \cap R_{1}^{p q}\left(B_{r}\right)$. (see the proof of Lemma 19 of [4] for the other cases).

Let us consider the case $i=j=2$. Assume by contradiction that a point $M$ of $E^{+}$exists in $R_{2}^{p q}\left(N_{A_{r} B_{r}}\right)$. By Lemma 3.11 $M \sim A \sim B$ and $M \in E_{0 p q}^{+}$. As $R_{2}^{p q}\left(\left\langle p\left(A_{r}\right), q\left(B_{r}\right)\right\rangle\right) \cap E^{+}=\emptyset$, we shall choose $M$ in such a way that $\lambda\left(R_{0}^{p q}(M) \cap E^{+}\right)>\mu$ and $\lambda\left(R_{2}^{p q}(M) \cap E^{+}\right) \leq \frac{\mu}{2}$. Since $F^{+}$and $F^{-}$have the same X-rays in directions $p, q$, we deduce that

$$
\lambda\left(R_{0}^{p q}(M) \cap E^{+}\right)+\lambda\left(R_{1}^{p q}(M) \cap E^{+}\right)=\lambda\left(R_{0}^{p q}(M) \cap E^{-}\right)+\lambda\left(R_{1}^{p q}(M) \cap E^{-}\right)
$$

and

$$
\lambda\left(R_{2}^{p q}(M) \cap E^{+}\right)+\lambda\left(R_{1}^{p q}(M) \cap E^{+}\right)=\lambda\left(R_{2}^{p q}(M) \cap E^{-}\right)+\lambda\left(R_{1}^{p q}(M) \cap E^{-}\right) .
$$


Thus, we obtain

$\lambda\left(R_{0}^{p q}(M) \cap E^{-}\right)=\lambda\left(R_{2}^{p q}(M) \cap E^{-}\right)+\lambda\left(R_{0}^{p q}(M) \cap E^{+}\right)-\lambda\left(R_{2}^{p q}(M) \cap E^{+}\right)>\frac{\mu}{2}$,

contradicting that $M \in E_{0 p q}^{+}$.

The cases $i=j=1$ and $i=j=3$ are similar with moreover the use of Lemma 3.10 to finally prove that $A_{r} \sim B_{r}$.

The special cases $r=p, r=q$ can also be easily checked.

Let $\mathcal{C}_{i p q}^{+}$be the set of equivalence classes on $E_{i p q}^{+}, \mathcal{C}^{+}=\cup_{(i, p, q) \in \mathcal{A}_{\mathcal{D}}} \mathcal{C}_{i p q}^{+}$and $\mathcal{C}=\mathcal{C}^{+} \cup \mathcal{C}^{-}$.

For every class $C \in \mathcal{C}^{+}$and direction $r \in \mathcal{D}$ a class $(C)_{r} \in \mathcal{C}^{-}$exists, and $C$ and $(C)_{r}$ have the same X-rays. If $C_{1}$ and $C_{2}$ are any two classes, we say that $C_{1}<_{p} C_{2}$ if $p\left(M_{1}\right)<p\left(M_{2}\right)$ for all $M_{1} \in C_{1}$ and $M_{2} \in C_{2}$.

Lemma 3.14 For any $(i, p, q) \in \mathcal{A}_{\mathcal{D}}$, the class $\mathcal{C}_{i p q}$ is non-empty.

Proof. Suppose $i=0$ and suppose that $\mathcal{C}_{0 p q}$ is empty. This means that for every class $C$ and point $M$ we have: $\left(C \cap R_{0}^{p q}(M)\right) \cap E^{-} \neq \emptyset$. Therefore $C_{p}<_{q} C$ or $C_{q}<_{p} C$ otherwise $C \subset \mathcal{C}_{0 p q}$. Suppose that $C_{q}<_{p} C$. Then, consider the sequence of classes defined by $C_{0}=C, C_{n+1}=\left(\left(C_{n}\right)_{q}\right)_{p}$. No class of the sequence is in $\mathcal{C}_{0 p q}$, and hence $C_{n+1}<_{p} C_{n}$ and $C_{n+1}<_{q} C_{n}$. So, the sequence consists of an infinite number of classes having the same area. This is not possible, and hence $\mathcal{C}_{0 p q}$ is non-empty.

Thanks to this lemma we can choose one class $C_{i, p, q}$ in each $\mathcal{C}_{i, p, q}$. Let $\mathcal{E}$ be the smallest set of the classes which contains all the $C_{i, p, q}$ and closed by the relations $C \mapsto C_{p}$. (This construction is essentially the same as in the proof of $[8$, Lemma 4].)

Lemma 3.15 The set $\mathcal{E}$ is finite.

Proof. Any class of $\mathcal{E}$ is Lebesgue-measurable because it is open and bounded. Let $\mathcal{E}^{\prime}$ be the subset of $E$ of the classes which area is equal to the area of one $C_{i, p, q}$. The set $\mathcal{E}^{\prime}$ is also closed by the relations $C \mapsto C_{p}$, so by minimality of $\mathcal{E}$ we have $\mathcal{E}=\mathcal{E}^{\prime}$. So if $\mathcal{E}$ was infinite then it $E$ would have an infinite area which is impossible because $E$ is a body.

Lemma 3.16 The classes of $\mathcal{E}$ can be ordered such that the corresponding sequence of their gravity-centers is a $\mathcal{D}$-sequence.

Proof. The proof is exactly the same as in Lemmas 21,22,24,25 of [4] after replacement of $\mathcal{C}$ by $\mathcal{E}$. 
This last lemma proves the implication (iii) $\Rightarrow$ (iv) of Theorem 3.4. So Theorem 3.4 is totally proved.

\section{Acknowledgement}

We wish to thank Paolo Gronchi for some useful discussions.

\section{References}

[1] Brunetti, S. and A. Daurat, An algorithm reconstructing convex lattice sets, Theoret. Comput. Sci. 304 (2003), 35-57.

[2] Brunetti, S. and A. Daurat, Stability in discrete tomography: Some positive results, Discrete Appl. Math. 147 (2005), 207-226.

[3] Brunetti, S., A. Daurat and A. Del Lungo, Approximate X-rays reconstruction of special lattice sets, Pure Math. Appl. 11 (2000), 409-425.

[4] Daurat, A., Determination of Q-convex sets by X-rays, Theoret. Comput. Sci. 332 (2005), 19-45.

[5] Fink, E. and D. Wood, Fundamentals of restricted-orientation convexity, Inform. Sci. 92 (1996), 175-196.

[6] Gardner, R. J., "Geometric Tomography," Encyclopedia of Mathematics and its Applications, Cambridge University Press, 1995.

[7] Gardner, R. J. and P. Gritzmann, Discrete tomography: determination of finite sets by X-rays, Trans. Amer. Math. Soc. 349 (1997), 2271-2295.

[8] Gardner, R. J. and P. McMullen, On Hammer's X-ray problem., J. Lond. Math. Soc., II. Ser. 21 (1980), 171-175.

[9] Giering, O., Bistimmung von Eibereichen und Eiköpern durch SteinerSymmetrisierungen, Bayer. Akad. Wiss. Math.-Natur. Kl. (1962), 225-253.

[10] Hammer, P., Problem 2, in: Convexity, Proceedings of Symposia in Pure Mathematics VII, American Mathematical Society, 1963 498-499.

[11] Herman, G. T. and A. Kuba, editors, "Discrete tomography," Appl. Numer. Harmon. Anal., Birkhäuser, 1999.

[12] Kölzow, D., A. Kuba and A. Volčič, An algorithm for reconstructing convex bodies from their projections, Disc. and Comp. Geom. 4 (1989), 205-237. 
[13] Volčič, A., Well-posedness of the Gardner-McMullen reconstruction problem, in: Measure theory, Oberwolfach 1983 (Oberwolfach, 1983), Lecture Notes in Math. 1089, Springer, 1984 199-210.

[14] Volčič, A., Tomography of convex bodies and inscribed polygons, Ricerche Mat. 36 (1987), 185-192. 